\title{
High-intensity interval training-induced hypertrophy in gastrocnemius muscle via improved IGF-I/Akt/FoxO and myostatin/Smad signaling pathways in rats
}

\author{
SOHEIL BIGLARI* (D, ALIREZA GHARDASHI AFOUSI (1), \\ FARNOOSH MAFI (D) and FATEMEH SHABKHIZ (1)
}

Department of Exercise Physiology, Faculty of Physical Education and Exercise Sciences, University of Tehran, Tehran, Islamic Republic of Iran

Received: November 26, 2019 • Accepted: April 4, 2020

Published online: July 07, 2020

(c) 2020 Akadémiai Kiadó

\begin{abstract}
Objective: It has been shown that high-intensity interval training (HIIT) leads to skeletal muscle hypertrophy; however, its mechanisms of cellular and molecular regulation are still unclear. The purpose of this study was to investigate the effect of HIIT on muscle hypertrophy and major signal transduction pathways. Design: 12 male rats were randomly divided into two groups: control and HIIT. The exercise group performed 30-min HIIT in each session $\left(5 \times 4\right.$-min intervals running at $85-95 \% \mathrm{VO}_{2 \max }$ separated by 2 -min active rest at $\left.55-60 \% \mathrm{VO}_{2 \max }\right), 3$ days/week for 8 weeks. Muscle fiber cross-sectional area (CSA) and the expression of signal transduction pathway proteins were determined in the gastrocnemius muscle. Results: In the HIIT group, the expression of IGF-I, IGF-IR Akt, p-Akt, AMPK $\alpha$, p-AMPK $\alpha$ and follistatin increased significantly, whereas a significant decrease was observed in the expression of FoxO1, p-FoxO1, myostatin, ActRIIB, Smad2/3 and $\mathrm{p}$-Smad2/3 $(P<0.05)$. However, there were no significant differences between the HIIT and control groups in the expression of mTOR, p-mTOR, P70S6K, and p-P70S6K $(P>0.05)$. In addition, CSA and gastrocnemius muscle weight increased significantly in the HIIT group $(P<0.05)$. Conclusions: HIIT induced muscle hypertrophy by improving IGF-I/Akt/FoxO and myostatin/Smad signal transduction pathways.
\end{abstract}

\section{KEYWORDS}

insulin-like growth factor I, follistatin, myostatin, cross-sectional area, muscle growth

\footnotetext{
* Corresponding author. Department of Exercise Physiology, Faculty of Physical Education and Exercise Sciences, University of Tehran, 16th Azar St., Enghelab Sq., Tehran, Islamic Republic of Iran. Tel.: +98 9183318578 , E-mail: s.biglari@ut.ac.ir
} 


\section{INTRODUCTION}

Muscle hypertrophy is characterized by a growth in muscle mass due to increased protein synthesis in muscle fibers. Several mechanisms regulate protein synthesis and myofiber growth; however, the major pathways of muscle growth regulation appear to be two signal transduction pathways: insulin-like growth factor-I/Akt/mammalian target of rapamycin (IGF-I/Akt/mTOR) and myostatin/Smad [1].

Increased protein synthesis induced by activation of the IGF-I/Akt/mTOR signal transduction pathway leads to hypertrophy [2]; one of the main factors in this signaling pathway is IGF-I, which stimulates muscle fiber growth by activating an intracellular cascade [3]. In addition, the IGF-I/Akt pathway can inhibit translocation of the forkhead box $\mathrm{O}$ (FoxO) transcription factor, which plays an important role in protein degradation [4]. Unlike the IGF-I/ Akt/mTOR pathway, the myostatin/Smad signaling pathway is a potent negative regulator of skeletal muscle growth [5]. Myostatin functions are influenced by interactive factors such as follistatin, which is known to be the most powerful antagonist of myostatin. By binding to the myostatin receptor (activin IIb), follistatin inhibits myostatin cellular function [6].

High-intensity interval training (HIIT) is a unique training model consisting of alternating periods of intensive exercise separated by inactive or active rest periods (low-intensity exercise). HIIT is a powerful strategy for structural and functional changes in skeletal muscle. The changes induced by HIIT are somewhat similar to what occurs following traditional endurance training: increases in mitochondrial biogenesis, metabolic enzyme activity, and oxidative capacity of muscle fibers [7]. It was recently shown that unlike traditional endurance training, HIIT leads to skeletal muscle hypertrophy in both trained and untrained healthy participants [8]. In addition, two studies found that muscle protein synthesis increased following HIIT in older adults and healthy young participants $[8,9]$. Robinson et al. reported that 12 weeks of HIIT in young and older adults led to significant increases in the transcriptional and translational regulation of muscle growth and mitochondrial pathway activity [8]. However, the mechanism responsible for the effect of HIIT on skeletal muscle hypertrophy is not fully known.

The repetition of high loads of work in HIIT over a short time may affect skeletal muscle hypertrophy by stimulating the expression of muscle growth factors [10]. However, muscle growth factor responses to HIIT over long periods are not well understood. Therefore, the purpose of the present study was to investigate the effect of 8 weeks of HIIT on muscle mass, cross-sectional area (CSA) and the expression of factors such as IGF-I, Akt, mTORC1, P70S6K, FoxO, myostatin, follistatin, Smad2/3, and AMP-kinase (AMPK) in the gastrocnemius muscle of male rats.

\section{MATERIALS AND METHODS}

\section{Animals}

Twelve male Sprague-Dawley rats (mean weight 200-250 g, 10 weeks old) were obtained from the animal laboratory of the Pasteur Institute of Iran. The animals were kept under standard housing conditions for laboratory animals (12-h light-dark cycle, average temperature $22 \pm 2{ }^{\circ} \mathrm{C}$ ) at the animal facility of the University of Tehran (Tehran, Iran). Rats were fed rodent pellet diet 
(diet \#5001, Purina Mills, Richmond, IN) and given free access to water. In this study, 12 rats were randomly divided into two groups: HIIT $(n=6)$ and control $(n=6)$. This study was approved by the Animal Ethics Committee of the University of Tehran (IR.UT.Rec.1396012) and performed according to the guidelines and instructions of the National Institutes of Health Guide for the Care and Use of Laboratory Animals (NIH publications No. 8023, revised 1978).

\section{Exercise test and exercise training}

The rats were familiarized with the treadmill by slow walking at a pace of $5 \mathrm{~m} / \mathrm{min}$ for $5 \mathrm{~min}$ in 5 sessions. Then exercise capacity tests were done 2 days before the beginning of the intervention and 2 days after the last exercise session at the end of week 8 . The exercise test started with 10 $\min$ at $10 \mathrm{~m} / \mathrm{min}$, and then the treadmill band velocity was increased by $0.03 \mathrm{~m} / \mathrm{s}$ every $2 \mathrm{~min}$ until the animals were unable to run further. At the end of the exercise test, maximum treadmill speed was record as the maximum running speed for each rat. In accordance with a previous study by Hoydal et al. $\mathrm{VO}_{2 \max }$ (intensity of exercise training) was calculated with the formula: $y=162 x-1\left(y=\mathrm{VO}_{2} \mathrm{ml} / \mathrm{kg} / \mathrm{min}, x=\right.$ running speed $\left.\mathrm{m} / \mathrm{s}\right)$ [9].

This protocol was used 3 days/week for 8 weeks with a small animal treadmill (Danesh Salar Iranian, Tehran, Iran), which included 5 min of warm-up at an intensity of $40-50 \% \mathrm{VO}_{2 \max }$ and $30 \mathrm{~min}$ of interval training. Each interval consisted of 4-min high intensity running (approximately $85-90 \% \mathrm{VO}_{2 \max }$ ) and 2-min active recovery (approximately $50-60 \% \mathrm{VO}_{2 \max }$ ). The training session ended with a 5 -min cool-down at $40-50 \% \mathrm{VO}_{2 \max }$ [10]. Training intensity was adjusted over time based on previous studies, running pace, and $\mathrm{VO}_{2 \max }$. Therefore the intensity was increased by $0.02 \mathrm{~m} / \mathrm{s}$ every week [9].

\section{Body weight and muscle sample collection}

Initial and final body weights were recorded in grams before the first session of the HIIT protocol and $48 \mathrm{~h}$ after the last training session. Forty-eight hours after the last training session, the animals were anesthetized with an intraperitoneal injection containing xylazine solution (10 $\mathrm{mg} / \mathrm{kg}$ body weight) and were euthanized with ketamine (90 $\mathrm{mg} / \mathrm{kg}$ body weight). Immediately after the rats were killed, their gastrocnemius muscles were excised, any connective tissue and fat were removed, and free-fat gastrocnemius muscle mass was weighed on a digital scale. Then the muscle samples were frozen in liquid nitrogen and stored at $-80{ }^{\circ} \mathrm{C}$ until analysis. The muscle was divided into two parts, which were used for histological and western blot analysis. Accordingly, one part of the muscle was placed in $4 \%$ paraformaldehyde for histological evaluation.

\section{Histological evaluation}

After dissection, the gastrocnemius muscle was rapidly fixed in 4\% paraformaldehyde for $24 \mathrm{~h}$. Then muscle tissues were embedded in paraffin and mounted on an ultrathin semiautomatic microtome (Dako, Tokyo, Japan) [11]. After fixation, serial transverse sections 8-10 mm thick were mounted on a glass slide and allowed to dry for $1 \mathrm{~h}$. Then the slides were stained with toluidine blue-1\% borax. Images of 5 different regions were obtained with a light microscope (Cxl, Labomed, Fremont, California, USA) equipped with a digital camera (Sony DSC S75, Tokyo, Japan). The CSAs of 50 random fibers were chosen from each picture for measurement 
with an image analysis system software (version 4.1.1.0; Digimizer, Belgium). A total of 300 muscle fibers per animal were used for CSA determinations.

\section{Enzyme-linked immunosorbent assay (ELISA)}

For ELISA, frozen samples of the medial gastrocnemius muscle were dissolved in lysis buffer containing $50 \mathrm{mM}$ Tris- $\mathrm{HCl}(\mathrm{pH} 7.4), 150 \mathrm{mM} \mathrm{NaCl}, 2 \mathrm{mM}$ EDTA, 1\% NP-40, and 10\% glycerol with a protease inhibitor cocktail tablet (Roche Diagnostics), and were homogenized and incubated on ice for $20 \mathrm{~min}$. The lysates were then centrifuged at $8,000 \mathrm{rpm}$ for $1 \mathrm{~h}$ at $4{ }^{\circ} \mathrm{C}$ to collect the supernatant for protein assay by IGF-I and IGF-IR (LifeSpan BioSciences, Seattle, WS, USA) according to the manufacturer's protocols. All samples were assayed in duplicate. Intra- and inter-assay coefficients of variation were 5 and $4 \%$ respectively for IGF-I, and 6 and $8 \%$ for IGF-IR.

\section{Western blotting}

Western blotting was used to quantify the amount of Akt, mTORC1, P70S6K, myostatin, follistatin, Smad 2/3, FoxO, ActRIIB, and AMP-kinase in the rat gastrocnemius muscle. First, 100 mg of medial gastrocnemius muscle tissue was homogenized in $200 \mu$ lysis buffer (RIPA buffer) (Sigma Aldrich, 10X, Munich, Germany) by cold protease inhibitor (Sigma Aldrich s8820). The homogenized tissue was centrifuged at $1,200 \mathrm{rpm}$ for $20 \mathrm{~min}$ at $4{ }^{\circ} \mathrm{C}$. Then the supernatant was isolated and stored at $-20{ }^{\circ} \mathrm{C}$. The protein concentration in the homogenate was determined with the Bradford test. Equal amounts of protein $(25 \mu \mathrm{g})$ in each sample were loaded for $12.5 \%$ standard SDS-polyacrylamide gel electrophoresis and were transferred to polyvinylidene difluoride (PVDF) membranes (Merck Millipore, Darmstadt, Germany) using a wet system (Bio-Rad, Paris, France). The PVDF membranes were blocked overnight with a solution containing non-fat milk in TBS-T buffer $(100 \mathrm{mM}$ Tris- $\mathrm{HCl}, 0.9 \% \mathrm{NaCl}$, and $0.1 \%$ Tween 20 [pH 7.4]) and were then incubated for $3 \mathrm{~h}$ at room temperature with one of the following primary antibodies at a dilution of 1:1,000: Akt (EPR 16798, ab179463), mTOR (EPR390, ab134903), myostatin (ab 198337), ActRIIB (EPR10739), Smad2/3 (ERP19557-4, ab202445), phosphoSmad2/3 (T8, ab63399), follistatin (ab64490), beta Actin (ab8229) (all from Abcam, Cambridge, UK), P70S6K (9202 S), phospho-P70S6K (Ser371, 9208 S), phospho-Akt (Ser473, 5012 S), phospho-mTOR (Ser2448, 2971 S), AMPK $\alpha$ (2793 S), phospho-AMPK $\alpha$ (Thr172, 50081 S), FoxO1 (C29H4, 2880 S), and phospho-FoxO1 (Thr24, 2599 S) (all from Cell Signaling Technology, Danvers, USA). The membranes were washed with TBS-T buffer 3 times for 15 min and then incubated with the secondary antibody (goat anti-rabbit IgG HRP 1:10,000; ab97051) for 90 min at room temperature. Immunoreactivity was revealed by enhanced chemiluminescence (ECL) (Amersham Biosciences, Freiburg, Germany). The blots were exposed to X-ray film sensitive to blue light for 5-30 s. Quantitative analyses were carried out for monomeric band data using Image J software. In additional, the phosphorylated protein/total protein ratio was calculated with Image J software. All analyses were done by analysts who were blinded to the group allocations of the samples. The antibodies were obtained from the Histogenotech Pasargad Laboratory (Tehran, Iran). 


\section{Statistical analysis}

The Shapiro-Wilk test and the Levene's test were used to verify the normality of distribution and homogeneity of variance, respectively. Independent sample t-tests were used to compare the mean values in the HIIT and control groups. All data are presented as means \pm standard error of the mean (SEM). All statistical analyses were conducted using GraphPad Prism-5 statistical software (LaJolla, CA, USA). Statistical significance was set at $P \leq 0.05$.

\section{RESULTS}

\section{Effect of HIIT on body and gastrocnemius weight and CSA}

Compared to the pretest results, body weight increased after 8 weeks of HIIT; however, this increase was not significantly different between groups. Gastrocnemius muscle wet weight increased significantly compared to the control group after 8 weeks of HIIT $(P=0.049)$ (Table 1). In addition, CSA increased significantly in the HIIT group compared to the control group $(P=0.047)$ (Fig. 1A).

\section{Effect of HIIT on mTOR signal transduction pathway factors}

The ELISA results showed that protein levels of IGF-1 $(P=0.018)$ and IGF-1R $(P=0.041)$ increased significantly in the HIIT group compared to the control group after the 8-week intervention (Fig. $2 \mathrm{~A}$ and $\mathrm{B})$. Western blot analysis showed that the expression of Akt $(P=$ $0.028)$ and p-Akt $(P=0.045)$ increased significantly in the HIIT group compared to the control group (Fig. $3 \mathrm{~A}$ and $\mathrm{B}$ ). However, there were no significant changes in the expression of $\mathrm{mTOR}$ $(P=0.096)$, p-mTOR $(P=0.082)$, or P70S6K $(P=0.091)$, p-P70S6K $(P=0.089)$ proteins in the HIIT group compared to the control group (Fig. $3 \mathrm{C}$ and $\mathrm{F})$. The expression of FoxO1 $(P=$ $0.007)$ and p-FoxO1 $(P=0.003)$ after the 8-week intervention decreased in the HIIT group compared to the control group (Fig. $3 \mathrm{G}$ and $\mathrm{H})$. Moreover, AMPK $\alpha(P=0.082)$ and p-AMPK $\alpha$ $(P=0.082)$ expression increased significantly in rats that received HIIT compared to untrained animals (Fig. 3I and J).

\section{Effect of HIIT on myostatin-Smad pathway factors}

Myostatin expression in the HIIT group decreased significantly compared to the control group $(P<0.0001$, Fig. $4 A)$. In addition, the expression of myostatin receptor (ActRIIB) was significantly downregulated in the HIIT group $(P=0.045$, Fig. $4 \mathrm{~B})$. Smad $2 / 3$ are downstream of

Table 1. Body and gastrocnemius muscle wet weight in rats after HIIT

\begin{tabular}{lccl}
\hline & Control $(n=6)$ & HIIT $(n=6)$ & $P$ values \\
\hline Initial body weight $(\mathrm{g})$ & $225.5 \pm 3.37$ & $226.3 \pm 3.58$ & 0.870 \\
Final body weight $(\mathrm{g})$ & $275.9 \pm 2.81$ & $274.8 \pm 3.47$ & 0.820 \\
Gastrocnemius wet weight $(\mathrm{g})$ & $1.29 \pm 0.006$ & $1.31 \pm 0.008^{\star}$ & 0.049 \\
\hline
\end{tabular}

Data are expressed as the mean \pm SEM.

${ }^{\star} P \leq 0.05$ vs. controls. 



Fig. 1. Cross-sectional areas (CSA) of gastrocnemius muscle fibers. (A) Representative histological images with toluidine blue staining in the gastrocnemius muscle. Scale bar $=20 \mu \mathrm{m}$. All data are presented as the mean \pm SEM. ${ }^{\star} P \leq 0.05$ vs. controls

myostatin signaling; Smad2/3 $(P=0.006)$ and $\operatorname{Smad} 2 / 3$ phosphorylation $(P=0.002)$ were downregulated after 8 weeks of HIIT (Fig. 4C and D). In contrast, follistatin expression was significantly upregulated after the HIIT intervention $(P=0.002$, Fig. $4 \mathrm{E})$.

A
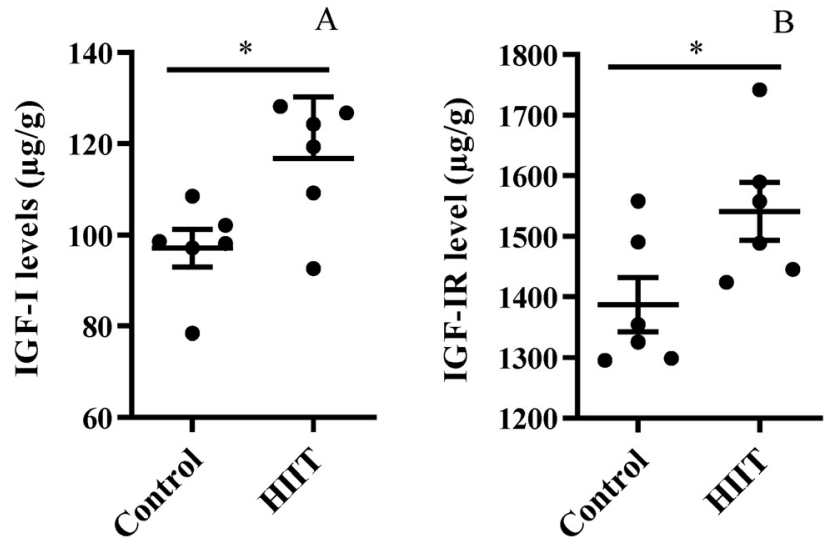

Fig. 2. Effect of high-intensity interval training (HIIT) on gastrocnemius muscle IGF-I. (A) and IGF-IR (B) levels determined with ELISA. All data are presented as the mean \pm SEM. ${ }^{\star} P \leq 0.05$ vs. controls 




Fig. 3. Effect of high-intensity interval training (HIIT) on the gastrocnemius Muscle Akt (A), p-Akt (B), mTOR (C) and p-mTOR (D), P70S6K (E), p-P70S6K (F), FoxO1 (G), p-FoxO1 (H) AMPK $\alpha$ (I) and $\mathrm{p}$-AMPK $\alpha(\mathrm{J})$ content with Western blotting. All data are presented as the mean $\pm \mathrm{SEM} .{ }^{\star} P<0.05,{ }^{\star *} \mathrm{P}<$ $0.01,{ }^{* *} P<0.001$ vs. controls 

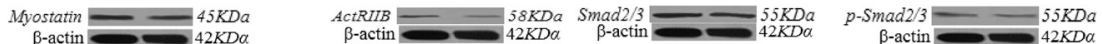

$\beta$-actin

-actin

A
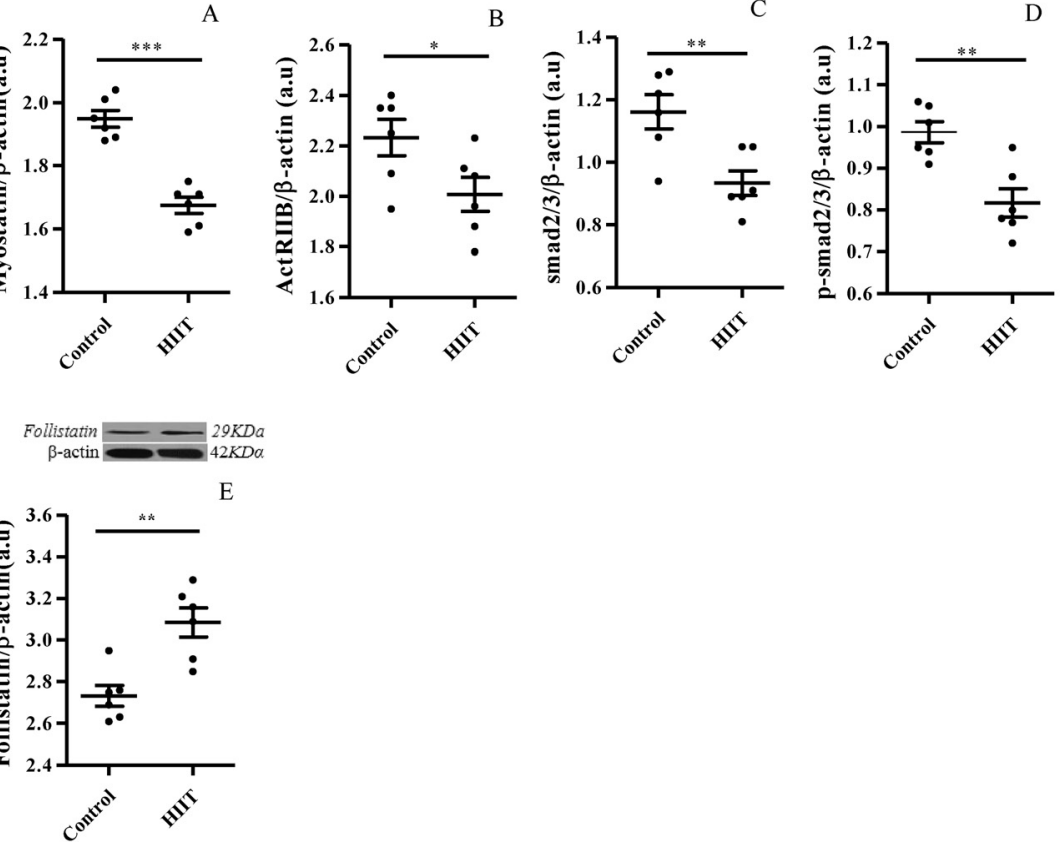

Fig. 4. Effect of high-intensity interval training (HIIT) on the gastrocnemius Muscle Myostatin (A), ActRIIB (B), smad2/3 (C), p-smad2/3 (D) and Follistatin (E) content with Western blotting. All data are presented as the mean \pm SEM. ${ }^{\star} P<0.05,{ }^{*} P<0.01,{ }^{* *} P<0.001$ vs. controls

\section{DISCUSSION}

The main finding of this study was the significant increase in skeletal muscle hypertrophy through IGF-I/Akt/FoxO and myostatin/Smad signal transduction pathways after long-term HIIT. Among the beneficial effects of HIIT was an increase in the activity of factors such as Akt, p-Akt, AMPK $\alpha$, p-AMPK $\alpha$, and follistatin protein expression; in addition, the intervention led to decreased expression of factors such as FoxO1, p-FoxO1, myostatin, ActRIIB, Smad2/3 and p-Smad2/3. However, no significant changes were observed in mTOR, p-mTOR, or P70S6K, p-P70S6K in the gastrocnemius muscle of healthy rats with or without HIIT. The present results indicate that the tissue protein content of IGF-I and IGF-IR was significantly enhanced following HIIT.

It is accepted that skeletal muscle growth is mediated by IGF-I, which acts through the PI3K/ Akt/mTOR/P70S6K pathway [12]. Our results showed that HIIT increased the content of IGF-I and its receptor IGF-IR in the gastrocnemius muscle in rats. Moreover, Act content and its phosphorylation levels also increased. Akt is downstream of the IGF-I signaling pathway, which positively modulates hypertrophy through activation of mTOR [12] and inhibition of FoxO1 by phosphorylation of these proteins [13]. However, in this study, muscle mTOR, p-mTOR and pP70S6K content did not change after HIIT. It has been shown that HIIT effectively stimulates 
mitochondrial biogenesis by enhancing AMPK signaling in skeletal muscle [14]. Furthermore, stimulation of AMPK was shown to inactivate mTOR through AMPK-mediated phosphorylation of both TSC2 and Raptor [15]. In this connection, it was also shown in both animal and human studies that a single bout of exercise and exercise training led to increased AMPK and the inhibition of mTOR $[16,17]$. In the present study, after long-term HIIT, the phosphorylation of AMPK $\alpha$ at Thr172 increased significantly.

It is known that $\mathrm{Akt} / \mathrm{FoxO}$ is one of the central signaling pathways that modulates skeletal muscle hypertrophy by inhibiting muscle atrophy [18]. One study found that treadmill exercise increased the expression of Akt and decreased levels of FoxO1 and FoxO1 phosphorylation, which resulted in skeletal muscle hypertrophy [19]. Our results indicated that long-term HIIT significantly activated Akt and decreased FoxO1 and FoxO1 phosphorylation; thus the Akt/ FoxO1 signal pathway may be involved in HIIT-induced muscle hypertrophy.

Our results also showed changes in the expression of myostatin and follistatin after long-term HIIT, which appeared to be associated with muscular hypertrophy after HIIT. Myostatin is known to be a negative regulator of skeletal muscle growth. It was shown that myostatin mutations in both animals and humans led to a remarkable increase in skeletal muscle mass and strength [19, 20]. Previous studies also demonstrated that the inhibition of myostatin expression after exercise training resulted in skeletal muscle hypertrophy [21-23]. Tang et al. have shown that endurance training inhibited mRNA and the expression of myostatin and its receptor ActRIIB [19]. In consonance with previous studies, our results showed that after long-term HIIT, the expression of myostatin and its receptor ActRIIB, and p-Smad2/p-Smad3, were significantly reduced.

By suppressing the inhibitory effects of myostatin on the differentiation of myogenic precursor cells and growth, and also by increasing protein synthesis, follistatin enhances skeletal muscle hypertrophy [11]. In one of the few studies designed to evaluate the effect of HIIT on myostatin, Elliott et al. reported that after 6 weeks of HIIT, the plasma levels of follistatin increased in sedentary participants [24]. In agreement with these previous findings, we show here that in untrained rats, long-term HIIT increased follistatin in the gastrocnemius muscle.

Recent studies have shown that HIIT increased protein synthesis, which resulted in muscle hypertrophy $[8,25]$. The hypertrophy in our HIIT group can be explained by the remarkable alterations in the myostatin/Smad and IGF-1/Akt/FoxO signaling pathways of skeletal muscle growth. After long-term HIIT, gastrocnemius muscle weight and CSA were significantly increased. In this connection, it was previously shown that myostatin/Smad signaling pathway inhibitors can increase muscle protein synthesis by enhancing the activity of the AKT/FoxO pathway [26, 27].

Some limitations of this study should be noted. First, although protein content and nitrogen balance are known to affect hypertrophy, we did not test energy, protein or nitrogen levels in our animals. Second, we did not test a transgenic model of hypertrophy in rats because we lacked access to transgenic animals. Further studies in transgenic animal models would shed further light of the effects of HIIT on muscle hypertrophy.

\section{CONCLUSION}

We investigated the effects of 8 weeks of HIIT on the IGF-I/Akt/mTOR, IGF1/Akt/FoxO and myostatin/Smad signaling pathways, and on CSA in the gastrocnemius muscle in rats. The HIIT intervention increased CSA by upregulating the IGF-1/Akt/FoxO pathway and downregulating 
the myostatin/Smad pathway. However, the IGF-1/Akt/mTOR signaling pathway had no effect on skeletal muscle growth after HIIT. To achieve a deeper understanding of the influence of HIIT on muscle growth mechanisms, future studies should investigate the effect of HIIT in other skeletal muscles and also in specific types of muscle fibers.

Funding: This research did not receive any specific grant from funding agencies in the public, commercial, or not-for-profit sectors.

Conflicts of interest: All authors declare no conflict of interest.

\section{ACKNOWLEDGMENTS}

We thank Histogenotech Pasargad Laboratory (Tehran, Iran) for their help with this study and K. Shashok (AuthorAID in the Eastern Mediterranean) for revising the use of English in the manuscript.

\section{REFERENCES}

1. Schiaffino S, Dyar KA, Ciciliot S, Blaauw B, Sandri M. Mechanisms regulating skeletal muscle growth and atrophy. FEBS J 2013; 280: 4294-314.

2. Bodine SC, Stitt TN, Gonzalez M, Kline WO, Stover GL, Bauerlein R, et al. Akt/mTOR pathway is a crucial regulator of skeletal muscle hypertrophy and can prevent muscle atrophy in vivo. Nat Cell Biol 2001; 3: 1014-19.

3. Rommel C, Bodine SC, Clarke BA, Rossman R, Nunez L, Stitt TN, et al. Mediation of IGF-1-induced skeletal myotube hypertrophy by PI (3) K/Akt/mTOR and PI (3) K/Akt/GSK3 pathways. Nat Cell Biol 2001; 3: 1009-13.

4. Calnan D, Brunet A. The foxo code. Oncogene 2008; 27: 2276.

5. McPherron AC, Lawler AM, Lee S-J. Regulation of skeletal muscle mass in mice by a new TGF-beta superfamily member. Nature 1997; 387: 83-90.

6. Lee Y-S, Lehar A, Sebald S, Liu M, Swaggart KA, Talbot CC, Jr, et al. Muscle hypertrophy induced by myostatin inhibition accelerates degeneration in dysferlinopathy. Hum Mol Genet 2015; 24: 5711-19.

7. Gillen JB, Gibala MJ. Is high-intensity interval training a time-efficient exercise strategy to improve health and fitness? Appl Physiol Nutr Metab 2014; 39: 409-12.

8. Robinson MM, Dasari S, Konopka AR, Johnson ML, Manjunatha S, Esponda RR, et al. Enhanced protein translation underlies improved metabolic and physical adaptations to different exercise training modes in young and old humans. Cell Metab 2017; 25: 581-92.

9. Høydal MA, Wisløff U, Kemi OJ, Ellingsen Ø. Running speed and maximal oxygen uptake in rats and mice: practical implications for exercise training. Eur J Cardiovasc Prev Rehabil 2007; 14: 753-60.

10. Afousi AG, Gaeini A, Rakhshan K, Naderi N, Azar AD, Aboutaleb N. Targeting necroptotic cell death pathway by high-intensity interval training (HIIT) decreases development of post-ischemic adverse remodelling after myocardial ischemia/reperfusion injury. J Cell Commun Signal 2019; 13: 255-67.

11. Winbanks CE, Weeks KL, Thomson RE, Sepulveda PV, Beyer C, Qian H, et al. Follistatin-mediated skeletal muscle hypertrophy is regulated by Smad3 and mTOR independently of myostatin. J Cell Biol 2012; 197: 997-1008. 
12. Schiaffino S, Mammucari C. Regulation of skeletal muscle growth by the IGF1-Akt/PKB pathway: insights from genetic models. Skelet Muscle 2011; 1: 4.

13. Xu M, Chen X, Chen D, Yu B, Huang Z. FoxO1: a novel insight into its molecular mechanisms in the regulation of skeletal muscle differentiation and fiber type specification. Oncotarget 2017; 8: 10662-74.

14. Cochran AJ, Percival ME, Tricarico S, Little JP, Cermak N, Gillen JB, et al. Intermittent and continuous highintensity exercise training induce similar acute but different chronic muscle adaptations. Exp Physiol 2014; 99: 782-91.

15. Gwinn DM, Shackelford DB, Egan DF, Mihaylova MM, Mery A, Vasquez DS, et al. AMPK phosphorylation of raptor mediates a metabolic checkpoint. Mol Cell 2008; 30: 214-26.

16. Drummond MJ, Dreyer HC, Pennings B, Fry CS, Dhanani S, Dillon EL, et al. Skeletal muscle protein anabolic response to resistance exercise and essential amino acids is delayed with aging. J Appl Physiol 2008; 104: 1452-61.

17. Thomson DM, Gordon SE. Impaired overload-induced muscle growth is associated with diminished translational signalling in aged rat fast-twitch skeletal muscle. J Physiol 2006; 574: 291-305.

18. Wimmer RJ, Liu Y, Schachter TN, Stonko DP, Peercy BE, Schneider MF. Mathematical modeling reveals modulation of both nuclear influx and efflux of Foxol by the IGF-I/PI3K/Akt pathway in skeletal muscle fibers. Am J Physiol Cell Physiol 2014; 306: C570-84.

19. Tang L, Zhang J, Zhao X, Li N, Jian W, Sun S, et al. Low-intensity pulsed ultrasound promotes exerciseinduced muscle hypertrophy. Ultrasound Med Biol 2017; 43: 1411-20.

20. Camporez J-PG, Petersen MC, Abudukadier A, Moreira GV, Jurczak MJ, Friedman G, et al. Anti-myostatin antibody increases muscle mass and strength and improves insulin sensitivity in old mice. Proc Natl Acad Sci U.S.A 2016; 113: 2212-7.

21. Ko IG, Jeong JW, Kim YH, Jee YS, Kim SE, Kim SH, et al. Aerobic exercise affects myostatin expression in aged rat skeletal muscles: a possibility of antiaging effects of aerobic exercise related with pelvic floor muscle and urethral rhabdosphincter. Int Neurourol J 2014; 18: 77-85.

22. Mosler S, Relizani K, Mouisel E, Amthor H, Diel P. Combinatory effects of siRNA-induced myostatin inhibition and exercise on skeletal muscle homeostasis and body composition. Physiol Rep 2014; 2: e00262.

23. Tang L, Luo K, Liu C, Wang X, Zhang D, Chi A, et al. Decrease in myostatin by ladder-climbing training is associated with insulin resistance in diet-induced obese rats. Chin Med J (Engl) 2014; 127: 2342-9.

24. Elliott BT, Herbert P, Sculthorpe N, Grace FM, Stratton D, Hayes LD. Lifelong exercise, but not short-term high-intensity interval training, increases GDF11, a marker of successful aging: a preliminary investigation. Physiol Rep 2017; 5: e13343.

25. Bell KE, Séguin C, Parise G, Baker SK, Phillips SM. Day-to-day changes in muscle protein synthesis in recovery from resistance, aerobic, and high-intensity interval exercise in older men. J Gerontol A Biol Sci Med Sci 2015; 70: 1024-9.

26. Allen DL, Unterman TG. Regulation of myostatin expression and myoblast differentiation by FoxO and SMAD transcription factors. Am J Physiol Cell Physiol 2007; 292: C188-99.

27. Sartori R, Milan G, Patron M, Mammucari C, Blaauw B, Abraham R, et al. Smad2 and 3 transcription factors control muscle mass in adulthood. Am J Physiol Cell Physiol 2009; 296: C1248-57. 